\title{
FORMATION AND GROWTH OF MICRO AND MACRO BUBBLES ON COPPER-GRAPHITE COMPOSITE SURFACES
}

\author{
David F. Chao ${ }^{1}$, John M. Sankovic ${ }^{2}$, and Brian J. Motil ${ }^{3}$ \\ NASA Glenn Research Center at Lewis Field, Cleveland, OH 44135 \\ and \\ Nengli Zhang ${ }^{4}$ \\ Ohio Aerospace Institute, Cleveland, OH 44142
}

\begin{abstract}
Micro scale boiling behavior in the vicinity of graphite micro-fiber tips on the coppergraphite composite boiling surfaces is investigated. It is discovered that a large number of micro bubbles are formed first at the micro scratches and cavities on the copper matrix in pool boiling. In virtue of the non-wetting property of graphite, once the growing micro bubbles touch the graphite tips, the micro bubbles are sucked by the tips and merged into larger micro bubbles sitting on the tips. The micro bubbles grow rapidly and coalesce to form macro bubbles, each of which sitting on several tips. The growth processes of the micro and macro bubbles are analyzed and formulated followed by an analysis of bubble departure on the composite surfaces. Based on these analyses, the enhancement mechanism of the pool boiling heat transfer on the composite surfaces is clearly revealed. Experimental results of pool boiling heat transfer both for water and Freon-113 on the composite surfaces convincingly demonstrate the enhancement effects of the unique structure of $\mathrm{Cu}-\mathrm{Gr}$ composite surfaces on boiling heat transfer.
\end{abstract}

\section{Nomenclature}

$=$ micro bubble diameter

$=$ macro bubble diameter

$=$ graphite fiber diameter

$=$ pressure

$=$ pressure difference

$=$ second principal radius of curvature of the vapor-liquid interface

$=$ first principal radius of curvature of the vapor-liquid interface

$=$ contracting rate of bubble throat radius

$=$ distance between coalescence plane and fiber tip

$=$ time

$=$ volume of microlayer in a micro bubble unit

$=$ vapor velocity

$=$ volume fraction of the graphite fiber in the composite material

$=$ height of the fiber tip plateau

$=$ average thickness of microlayer

$=$ maximum thickness of microlayer

${ }_{1}^{1}$ Aerospace Engineer, Microgravity Division, Mail Stop 77-5.

${ }^{2}$ Manager, Radioisotope Power Systems Science Division, MS 142-2, AIAA Senior Member.

${ }^{3}$ Aerospace Engineer, Microgravity Division, MS 86-8, AIAA Member.

${ }^{4}$ Corresponding author, Senior Scientist, Department of Workforce Enhancement, MS 77-5. 


\begin{tabular}{|c|c|c|}
\hline$\rho$ & & density \\
\hline$\sigma$ & $=$ & surface tension \\
\hline \multicolumn{3}{|c|}{ Subscripts } \\
\hline$b$ & $=$ & bottom \\
\hline$d$ & $=$ & departure \\
\hline$l$ & $=$ & liquid \\
\hline$T$ & $=$ & top of bubble \\
\hline th & $=$ & throat \\
\hline$v$ & $=$ & vapor \\
\hline
\end{tabular}

\section{Introduction}

A $\mathrm{S}$ is well known, the nucleate boiling is a greatly effective heat transfer mode. Although over years researchers have experimentally and theoretically studied boiling process, there are no theories or literature yet that can exactly explain the underlying heat transfer mechanisms. However, an accepted physics is that bubble nucleation, growth and departure control the heat and mass transport rate. The basic understanding of boiling phenomena on plain surfaces brought about ideas modifying the process through changing properties of the working fluid or/and the boiling surface to increase the boiling heat transfer coefficient. Thome ${ }^{1}$ classified the enhancement techniques as passive and active techniques and considered that the passive techniques have the most practical importance. The passive techniques have been considered to include treated, rough, extended surfaces and surface tension devices. Fifteen years ago, a new passive technique, so called metal-graphite composite surfaces, was presented and studied. ${ }^{2}$ Since then a series of investigations have been performed to determine the enhancement effects of the composite surfaces on boiling heat transfer and their mechanism. ${ }^{3-7}$ It was disclosed that the nucleate pool boiling heat transfer in Freon-113 (a highly wetting liquids) on copper-graphite (Cu-Gr) composite surfaces is increased up to four to six times over on a pure copper surface. The critical heat flux (CHF) on metal-graphite heating surfaces have also been investigated $^{8,9}$ and a correlation equation for predicting CHF has been suggested. ${ }^{9}$ Liang and Yang ${ }^{10}$ tested pentane (another highly wetting liquid) nucleate pool boiling performance on both $\mathrm{Cu}-\mathrm{Gr}$ and aluminum-graphite (Al-Gr) composite surfaces with a fiber volume fraction of $50 \%$ and concluded that the presence of graphite fibers not only enhances overall boiling performance in developed region but also reduces hysteresis effects in the start up region which is important for the practical application of immersion cooling in the electronic industry. Chao ${ }^{11}$ et al conducted experiments on nucleate pool boiling both in a highly wetting liquid, Freon-113, and a moderately wetting fluid, water, on $\mathrm{Cu}-\mathrm{Gr}$ composite surfaces with different fiber volume fractions. The experimental results have shown that significant enhancement in pool boiling heat transfer performance in both highly wetting liquids and moderated wetting liquids like water occurs on $\mathrm{Cu}-\mathrm{Gr}$ composite surfaces.

The enhancement mechanisms of boiling on the composite surfaces are believed to be related with: 1) the higher thermal conductivity of micro-graphite fibers, 2) a large number of embryo cavities of appropriate size, and 3) the poorly-wetted property of graphite fiber tips. ${ }^{10}$ In order to clarify the physics of the enhancement, it is important to have an understanding of the processes of bubble formation, growth and departure.

For common solid surfaces, numerous models had been developed to predict bubble detachment diameters in pool boiling and were reviewed by Zeng et al. ${ }^{12}$ Many experiments have showed that the bubble detachment had been implemented by a necking process. ${ }^{13-17}$ Mitrovic $^{15,16}$ analyzed the movement of three-phase-line (TPL) and the formation of bubble neck during growth of the bubble. He considered evaporation at TPL tended to create a convex interface, leading to establish the Laplace-pressure, which pushed the liquid radially outward to form the bubble neck. Kandlikar and Steinke ${ }^{18}$ suggested that an intense evaporation at the TPL exerted a vapor recoil force pushing the interface into the liquid and consequently, a convex vapor-liquid interface was formed and developed to become the bubble neck. Although the bubble necking and broken-off processes were described by Mictrovic ${ }^{15}$ and Mori and Baines, ${ }^{17}$ but the dynamic process has not been properly analyzed. Recently, Zhang et al. ${ }^{19}$ have analyzed the detail dynamic process of the bubble growth and necking, and subsequently given a determination method of the bubble departure diameter. As for the case of boiling on $\mathrm{Cu}-\mathrm{Gr}$ composite surfaces, the bubble formation, growth, and departure dynamic processes have not been well investigated yet.

\section{Formation and Growth of Micro Bubbles}

Different from common metal surfaces, the metal-graphite composite surfaces are of their unique geometric and physical characteristics. The polished composite surfaces are smooth in macro scale but rough in micro scale. Yang ${ }^{5}$ investigated the surface structure through scanning electronic microscope (SEM). Direct top-view photomicrographs of a $\mathrm{Cu}-\mathrm{Gr}$ composite specimen with volume fraction of $50 \%$ graphite-fiber and fiber diameter of $8 \sim 10 \mu \mathrm{m}$ clearly 
display that the graphite fibers are embedded in the copper matrix perfectly without any gap or void, as shown in Fig. 1a. The photomicrographs also show that the fiber tips are rather smooth while the copper matrix surface is filled with a large number of microsized low-lying trenches and intermingled narrow grooves. A slightly tilted topview of the composite surface shows that the fiber tips bulge out of the copper matrix, surviving the polishing due to their pliability and tenacity, and form individual plateaus distributing evenly on the composite surfaces, as shown in Fig. $1 b$.

In contradistinction to the intuition, the preliminary experimental observations have found that the micro bubbles originate from the valleys between the fiber tip plateaus instead of the fiber tips ${ }^{5}$. It is obviously attributed to that plenty of potential nucleate sites are created by the microsized trenches and intermingled narrow grooves on the copper matrix surface. On the other hand, although the fiber tips may have higher temperature than the copper matrix surface, the relative smooth surface of the fiber tip plateaus hardly provide initial nucleate sites.

Once the growing micro bubbles touch the fiber tips, the micro bubbles tend to migrate to the tips from the copper matrix and firmly sit on the tips for the poor wetting character of the graphite and a large surface tension at the TPL. As the micro bubbles sitting on the fiber tips grow, the newly emerging embryo bubbles on the copper matrix would be swallowed by the micro bubbles at a touch, as shown in Fig. 2a. Both the rapid evaporation due to the higher temperature of the graphite fiber tips and the swallow of the emerging embryo micro bubbles speed up the growth of the micro bubbles on the fiber tips. The further growing micro bubbles inevitably coalesce with each other, as shown in Fig. 2b. The coalesced micro bubbles rapidly grow up and come into being micro mushrooms with their own microlayers forming a liquid network connected with bulk liquid under the coalesced micro bubbles, as shown in Figs. 2c and 2d.

As analyzed by Zhang et al, ${ }^{3}$ the micro bubbles coalesce at their maximum cross section. By reference to Fig. 2c, the micro bubble diameter at coalescence, $D$, is related to the fiber diameter, $d$, and the volume fraction of the graphite fiber in the composite material, $\alpha$, which is identical with the area fraction of the fiber tips in the composite surface, by the following equation:

$$
\pi d^{2} / 4=\alpha D^{2}
$$

The height of coalescence plane of the micro bubbles is regarded as the maximum thickness of the microlayer $\delta_{m}$. It can be seen from Fig. $2 \mathrm{~b}$ that $\delta_{m}=\Delta+\mathrm{S}$, where $\Delta$ is the height of the fiber tip plateau, estimated from Fig. $1 \mathrm{~b}$ at about $d / 30, S$ is the distance between coalescence plane and fiber tip, equaling $\sqrt{(D / 2)^{2}+(d / 2)^{2}}=d \sqrt{\pi /(4 \alpha)-1 / 2}$.

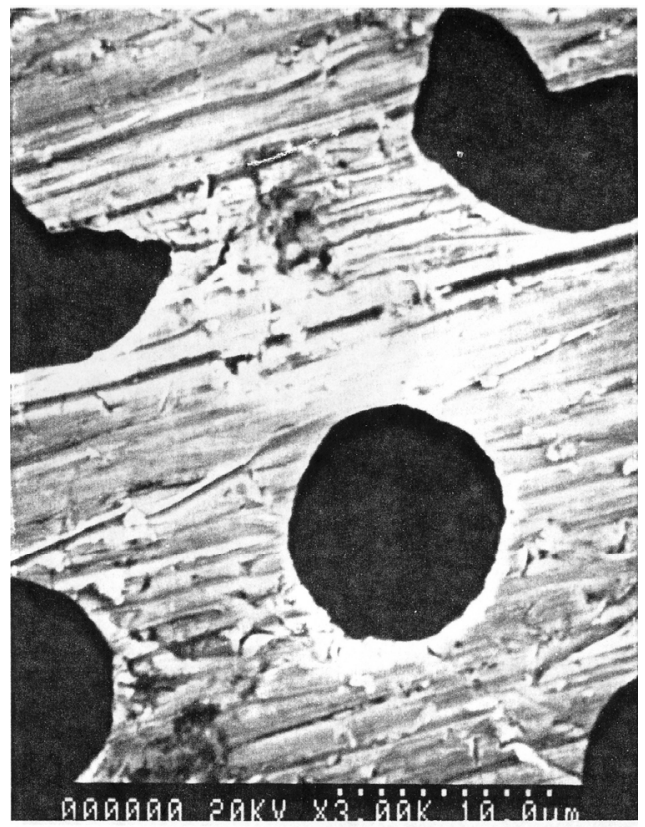

a)

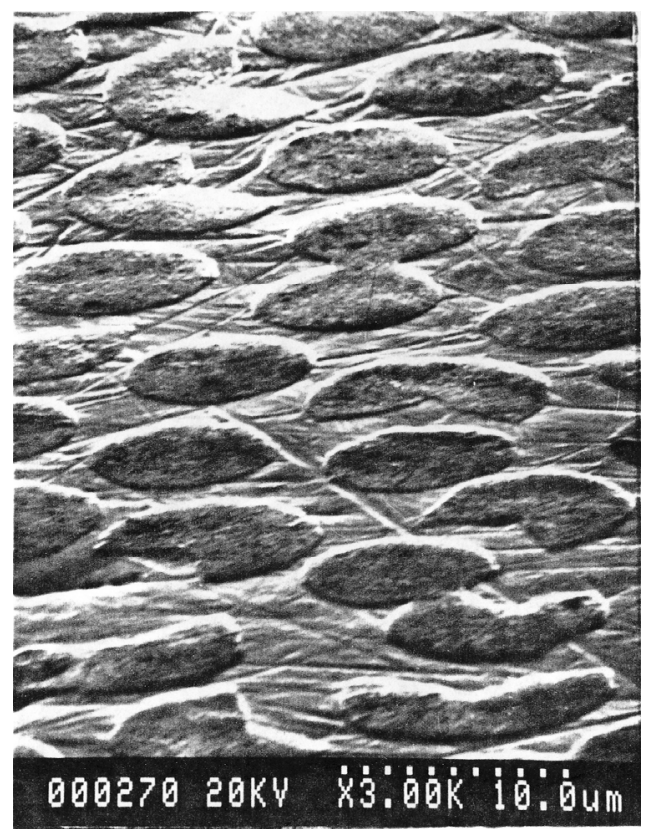

b)

Figure 1. Photomicrographs (3,000 times) of a $\mathrm{Cu}-\mathrm{Gr}$ composite surface with volume fraction of $50 \%$ graphite-fiber (8 10 $\mu \mathrm{m}$ diameter): a) direct top view; b) slightly tilted top view. 


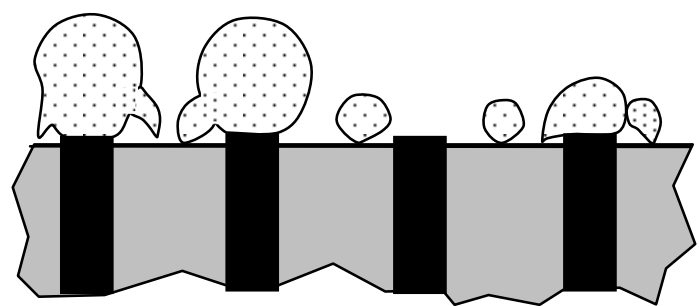

a)

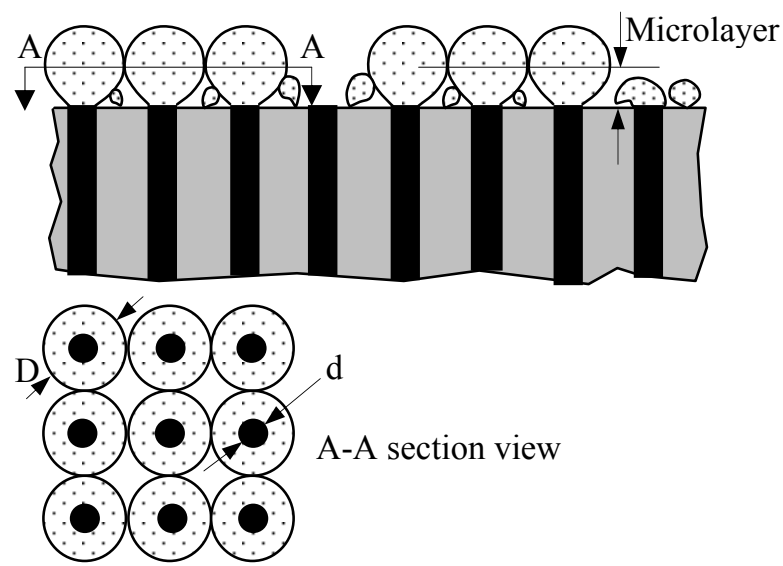

c)

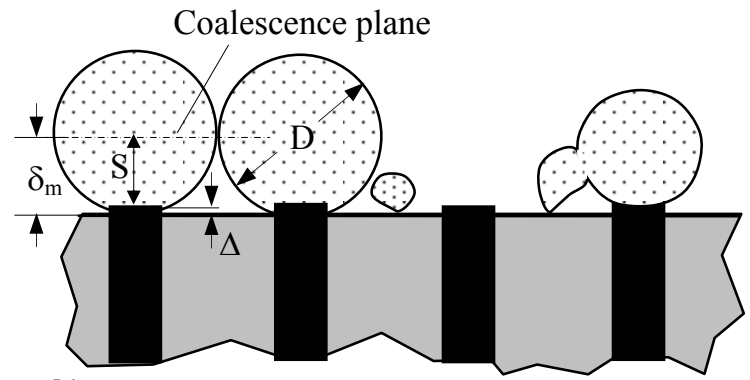

b)

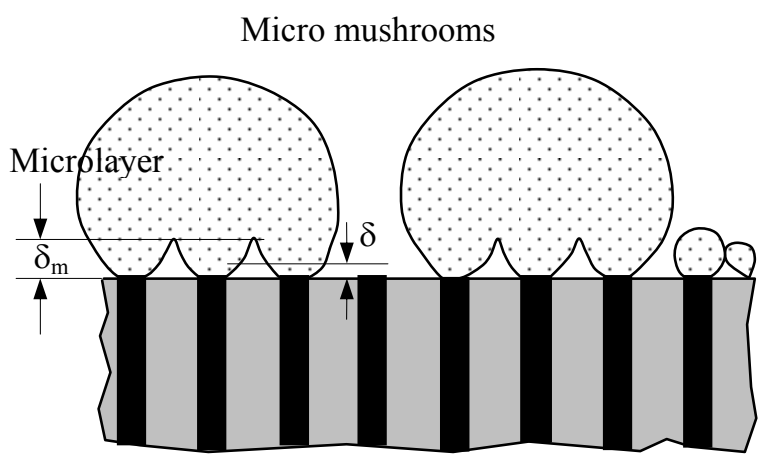

d)

Figure 2. Initiation and growth of micro bubbles: a) initiation and migration of micro bubbles; b) grow of micro bubbles firmly sitting on the fiber tips; c) coalescence of micro bubbles d) growth of coalesced micro bubbles and formation of micro-mushrooms.

Therefore,

$$
\delta_{m}=\frac{d}{2} \sqrt{\frac{\pi}{4 \alpha}-1}+\Delta
$$

Based on simple geometry, the volume of the microlayer in a micro bubble unit can be calculated by

$$
V_{m}=D^{2} \delta_{m}-\frac{1}{6} \pi S\left[\frac{3}{4} D^{2}+\frac{3}{4} d^{2}+S^{2}\right]-\frac{\pi d^{2}}{4} \Delta
$$

Idealizing the liquid volume as a column of constant cross section, the average thickness of microlayer, $\delta$, can be derived by $V_{m} /\left(D^{2}-\pi d^{2} / 4\right)$. As a result, the following expression can be obtained:

$$
\delta=\left[\frac{1-\pi / 6-\alpha / 3}{2(1-\alpha)} \sqrt{\frac{\pi}{4 \alpha}-1}+\frac{1}{30}\right] d
$$

Obviously, both the maximum and average thicknesses of the microlayer are determined by the volume fraction of graphite fiber in the composite $\alpha$ and the fiber diameter $d$. As mentioned above, $d=8 \sim 10 \mu \mathrm{m}$, simple calculation results for the maximum and average thicknesses of the microlayer at $d=$ $9 \mu \mathrm{m}$ are listed in Table 1 .

Table 1. Microlayer thicknesses at different values of $\alpha$

\begin{tabular}{lcccc}
\hline$\alpha$ & 0.1 & 0.25 & 0.5 & 0.75 \\
$\delta_{\mathrm{m}}(\mu \mathrm{m})$ & 12.08 & 6.89 & 3.70 & 1.28 \\
$\delta(\mu \mathrm{m})$ & 6.10 & 3.75 & 2.41 & 1.19
\end{tabular}


In boiling heat transfer, microlayers play an essential role. Thermal energy provided by the composite surface is first conducted through the microlayers and then delivered into the micro mushrooms as latent heat by evaporation at the microlayer surface. Because the microlayers are connected directly with the bulk liquid through liquid network, the evaporated liquid of the microlayers can immediately be replenished. It is the ample feed of liquid into the microlayers that enables the composite surfaces to have lower superheat at a given heat fluxes of boiling, i. e. enhancement of boiling heat transfer.

\section{Formation of a Macro Bubble}

The continually growing micro mushrooms inevitably coalesce and merge with each other to form a larger micro vapor mass, which is a macro bubble embryo, as shown in Fig. 3. When the bubble embryo is formed from several micro mushrooms, a macrolayer is created under the macro bubble embryo, connecting with the microlayers and bulk liquid through liquid network. As analyzed by Mitrovic ${ }^{15,16}$ and $\operatorname{Kandlikar}^{18}$ for a bubble growth, at the beginning of the bubble formation, the TPL of the bubble would move outwards to forms a convex vapor-liquid interface at the bubble bottom, and subsequently a bubble neck is created. The same process also occurs in the growth of a macro bubble embryo on the composite surfaces. As the macro bubble embryo grows, the TPL of the macrolayer moves towards liquid under the combination of high evaporation rate and capillary-pressure difference. Zhang et $\mathrm{al}^{19}$ analyzed this kind of TPL moving process in detail. In the case of a macro bubble embryo formed on the $\mathrm{Cu}-\mathrm{Gr}$ composite surfaces, the moving TPL would stop at next circles lined with the corresponding neighbor graphite fiber tips for the poor wettability of the graphite fiber, and consequently, convex vapor-liquid interfaces are created at the macro bubble embryo bottom both the inner and the outer TPLs, as shown in Fig. $3 \mathrm{~b}$. The macro bubble embryo rapidly grows up and finally forms a macro bubble with a neck being of a throat radius of $r_{t}$ and the bottom radius of $r_{b}$ whose value is estimated at $0.1 \sim 0.2 \mathrm{~mm}$, as shown in Fig. 4.

\section{Departure of a Macro Bubble}

The strong evaporation of liquid from the micro and macro layers is firmly supported by the ample liquid supply through the liquid network at the bubble bottom, and creates larger and larger negative pressure at the throat, i. e. $\Delta p_{t h}=p_{l, t h}-p_{v, t h}>0$, where $p_{l, t h}$ and $p_{v, t h}$ are the static pressures of liquid side and vapor side at the throat, respectively.

It should be noted that the static pressure difference varies along the bubble interface, as shown in Fig. 5. At the top of a departing bubble the static pressure difference $\Delta P_{T}$ can be expressed as $4 \sigma / \mathcal{D}_{d}$, where $\mathcal{D}_{d}$ is the bubble departure diameter, while the static pressure difference at the throat $\Delta P_{t h}$ is estimated by

\section{Coalescing micro mushrooms}

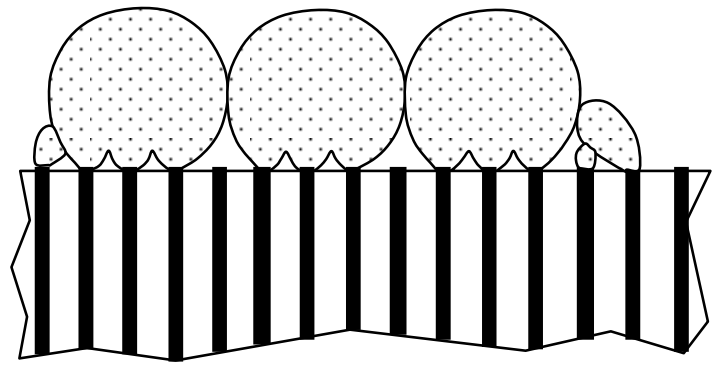

a)

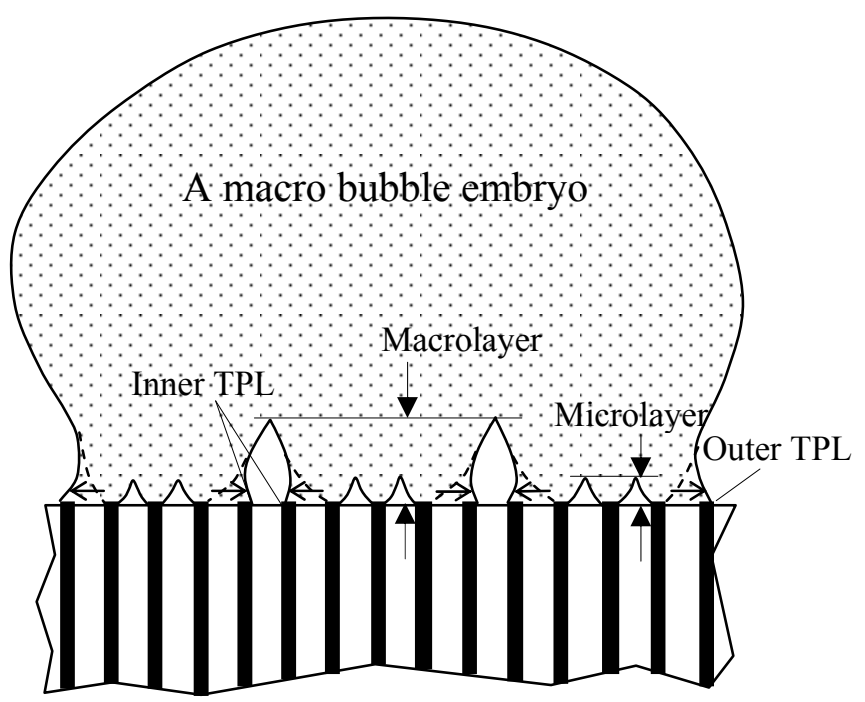

b)

Figure 3. Formation of a macro mushroom from coalesced micro mushrooms: a) coalescence of micro mushrooms; b) formation of a macro bubble embryo. 
A growing macro bubble

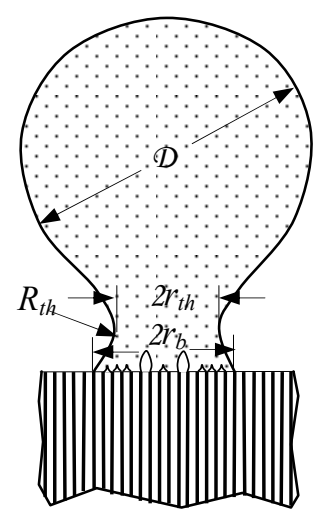

Figure 4. Formation of a macro bubble

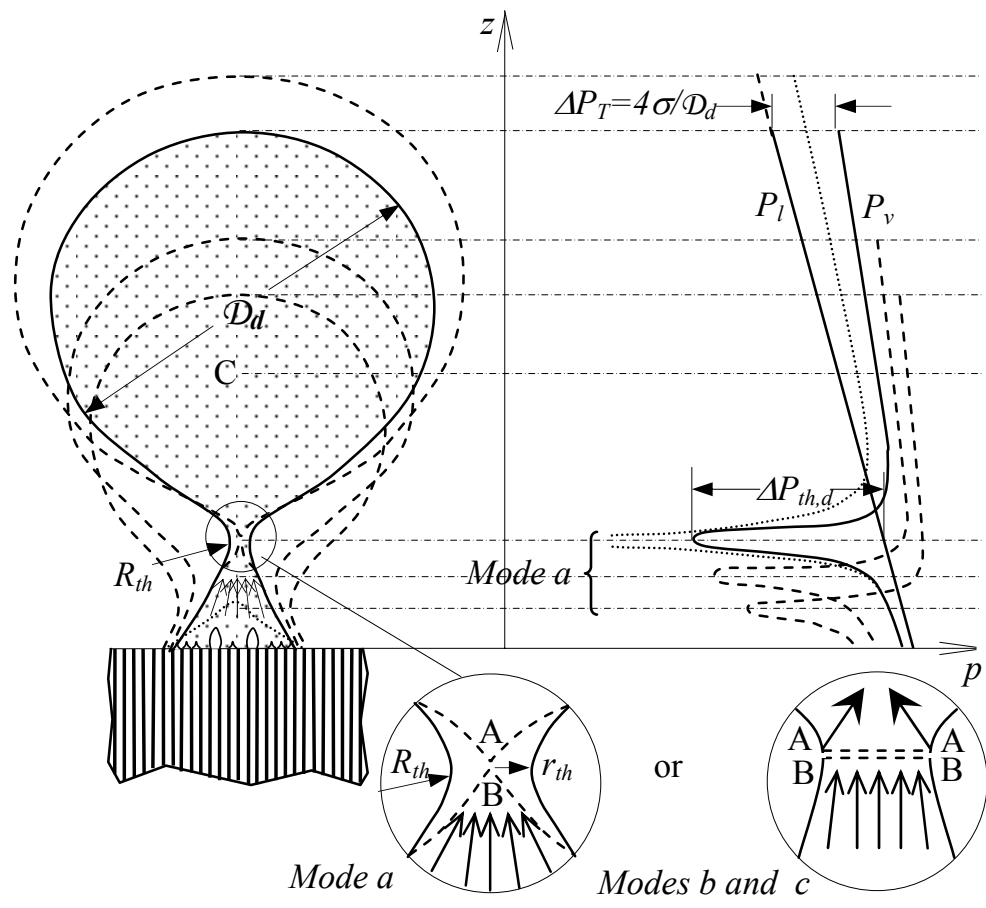

Figure 5. Growth, necking, and departure of a macro bubble.

$$
\Delta p_{t h}=\left(\rho_{v} W_{t h}^{2}-\rho_{l} \dot{r}_{t h}^{2}\right) / 2-\left(\sigma / r_{t h}-\sigma / R_{t h}\right)
$$

where $\rho_{v}$ and $\rho_{l}$ are the density of vapor and liquid, respectively, $W_{t h}$ is the vapor velocity at throat, $\dot{r}_{t h}$ is the contracting rate of the throat radius, i. e. liquid velocity at throat, $\sigma$ is the surface tension, $R_{t h}$ is the second principal curvature radius of the vapor-liquid interface at throat.

As analyzed by Zhang et al ${ }^{19}$ the bubble necking and departure have three possible modes :

$a$. If change rate of the static pressure difference at bubble throat is positive $\left(d\left(\Delta p_{t h}\right) / d t>0\right)$, the bubble neck would be broken off rapidly with a very short necking process.

$b$. If $d\left(\Delta p_{t h}\right) / d t=0$, the bubble neck continually and gently contracts before the neck is broken off.

$c$. For the case of $d\left(\Delta p_{t h}\right) / d t<0$, the bubble neck contracts to a certain diameter and then maintains it until the bubble grows big enough to be broken off at the throat.

In mode $a$, since $d\left(\Delta p_{t h}\right) / d t>0$, the static pressure difference $\Delta P_{t h}$ becomes larger and larger, and consequently, the throat is rapidly compressed to zero and then the neck is broken off, while in mode $b$, the static pressure difference $\Delta P_{t h}$ maintains a constant till the buoyancy of the bubble exceeds the surface tension force at the throat during the throat contracting process and so the neck is broken off at a finite throat diameter. However, in the mode $c, d\left(\Delta p_{t h}\right) / d t<0$, the $\Delta P_{t h}$ finally decreases to zero and the throat reduces to and then maintains a certain value till that the growing buoyancy exceeds the surface tension at the throat to break the neck off. For mode $a$, the $\Delta P_{t h}$ experiences a sudden change, from a certain value to an uncertain value when the throat diameter reduced to zero. As a result, the major portion of the bubble above the neck departures with a singular point at the broken throat (point A in the Fig. 5). For both modes $b$ and $c$, a singular circle line A-A is created at the broken neck of the departing bubble, at which a theoretical infinity of $\Delta P_{t h}$ occurs. This is the reason of occurrence of a liquid jet in the departure bubble. Of course, the corresponding singular point B in mode $a$ or circle singular line B-B in modes $b$ and $c$ is formed at the residual vapor portion sitting on the composite surface, as shown in Fig. 5, but the strong evaporation from the liquid network at the residual bubble bottom withstands the action of the sudden change of $\Delta P_{t h}$, and at the same time rapidly fosters and develops a new bubble. 


\section{Enhancement of Boiling Heat Transfer}

It is because the unique structure of $\mathrm{Cu}-\mathrm{Gr}$ composite surfaces that the strong evaporation in macro bubbles is supported by the ample liquid supply through the liquid network connecting the macrolayers and microlayers to bulk liquid, and consequently, much higher heat flux can be reached in the boiling heat transfer.

Based on the force balance at neck of a departing bubble, Chao et al ${ }^{11}$ have theoretically and experimentally revealed that higher vapor velocity produced by a larger local heat flux of boiling on the composite surface enables the bubbles to depart at smaller departure diameters, and consequently, results in an increased bubble departure frequency. This enhancement mechanism can successfully explain the experiment results of the enhanced boiling heat transfer both for F-113 and water. ${ }^{2,6,11}$

\section{Conclusions}

In pool boiling on $\mathrm{Cu}-\mathrm{Gr}$ composite surfaces, micro bubbles originate first from the valleys between the fiber tip plateaus instead of the fiber tips on the graphite tips, and then migrate to the tips. The growing micro bubbles sitting on the fiber tips coalesce and merge each other to produce macro bubble embryos with a liquid network consisting of micro- and macro-layers. As an important parameter, the microlayer thickness is analyzed and formulated.

It is the unique structure of $\mathrm{Cu}-\mathrm{Gr}$ composite surfaces that creates a liquid network connected to bulk liquid, from whom an ample supply of liquid firmly supports the strong evaporation at the surfaces of micro- and macrolayers and fosters and develops macro bubbles. During the formation process of a macro bubble, a convex-concave interface is created at the bubble bottom under the combination of high evaporation rate and capillary-pressure difference, and then developed to a neck with a narrower and narrower throat. It is the strong evaporation that produces larger and larger vapor velocity and creates a great negative pressure at the bubble throat which accelerates the bubble departure with smaller departure diameters and higher departure frequency, enhancing the boiling heat transfer.

\section{References}

${ }^{1}$ Thome, J. R., Enhanced Boiling Heat Transfer, New York: Hemisphere, 1990, Chapter 3.

${ }^{2}$ Yang, W. J., Takizawa, H. and Varable. D., “Augmented on Copper-Graphite Composite Surface,” International Journal Heat and Mass Transfer, Vol. 34, No.11, 1991, pp. 2751-2758.

${ }^{3}$ Zhang, N., Yang, W. J., and Yang. G. W., "Two-Tier Model for Nucleate Pool Boiling on Micro Configured Composite Surfaces," International communications in Heat and Mass Transfer. Vol. 19, No. 6, 1992, pp. 767-779.

${ }^{4}$ Yang, G. W., Yang, W. J., and Zhang, N., "Mechanisms of Nucleate Pool Boiling on Composite Surfaces," International. communications in Heat and Mass Transfer, Vol. 19, No. 6, 1992, pp. 781-790.

${ }^{5}$ Yang, G. W., "Micro- and Macro-phenomena in Nucleate Pool Boiling on Graphite-Copper Composite Materials," Ph.D. Dissertation, Department of Mechanical Engineering and Applied Mechanics, The University of Michigan, Ann Arbor, Michigan, 1995.

${ }^{6}$ Yang, G. W., Liang, H.-S., Yang, W. J., and Vrable. D. L., "Nucleate Pool Boiling on Micro Graphite-Copper Composite Surfaces," Journal Heat Transfer, Vol.118, No. 3, 1996, pp. 792-796.

${ }^{7}$ Liang, H.-S., "Nucleate Pool Boiling on Micro-Graphite-Fiber Composite Surfaces with Applications in Micro-Electronic Cooling," Ph.D. Dissertation, Department of Mechanical Engineering and Applied Mechanics, The University of Michigan, Ann Arbor, Michigan, 1997.

${ }^{8}$ Yang, W. J., and Zhang, N., "A Theoretical Treatment of Critical Heat Flux on Metal-Graphite Heating Surfaces," Proceedings of International ME'99 Congress \& Exposition, the ASME Heat Transfer Division, Vol. 2 (HTD-Vol. 364-2), 1999, pp.315-320.

${ }^{9}$ Zhang, N., Chao, D. F., and Yang. W. J., "Enhancements of Nucleate Boiling and Critical Heat Flux under Microgravity Conditions," Journal of Thermophysics and Heat Transfer. Vol. 15, No. 3, 2001, pp. 326-332.

${ }^{10}$ Liang, H.-S., and Yang, W. J., "Nucleate Pool Boiling Heat Transfer in a Highly Wetting Liquid on Micro-graphite-fiber Composite Surfaces," International Journal Heat and Mass Transfer, Vol. 41, No. 13, 1998, pp. 1993-2001.

${ }^{11}$ Chao, D. F., Zhang, N., and Yang, W. J., "Nucleate Pool Boiling on Copper-Graphite Composite Surfaces and Its Enhancement Mechanism," Journal of Thermophysics and Heat Transfer, Vol. 18, No. 2, 2004, pp. 236-242.

${ }^{12}$ Zeng, L. Z., Klausner, J. F., and Mei, R., "A Unified Model for the Prediction of Bubble Detachment Diameters in Boiling Systems - - I. Pool Boiling," International Journal Heat and Mass Transfer, vol. 36, No. 10, 1993, pp. 2261-2270.

${ }^{13}$ van Stralen, S.J.D., Cole, R., Sluyter, W.M., and Sohal, M.S., International Journal Heat and Mass Transfer, vol. 18, pp. 655-669, 1975.

${ }^{14}$ Nordmann, D., and Mayinger, F., "Temperatur, Druck und Wärmetransport in der Umgebung Kondensierender Blasen," VDI-Forschungsheft, Nr. 605, 1981.

${ }^{15}$ Mitrovic, J., "Formation of a Liquid Jet after Detachment of a Vapour Bubble," International Journal Heat and Mass Transfer, vol. 40, No.18, 1997, pp. 4309-4317. 
${ }^{16}$ Mitrovic, J., "The Flow and Heat Transfer in the Wedge-shaped Liquid Film Formed During the Growth of a Vapour Bubble," International Journal Heat and Mass Transfer, Vol. 41, No. 12, 1998, pp. 1771-1785.

${ }^{17}$ Mori, B. K., and Baines, W. D., "Bubble Departure from Cavities," International Journal Heat and Mass Transfer, vol. 44, No. 4, 2001, pp. 771-783.

${ }^{18}$ Kandlikar, S.G., and Steinke, M.E., "Contact Angles and Interface Behavior during Rapid Evaporation of Liquid on a Heated Surface," International Journal of Heat and Mass Transfer, Vol. 45, No. 2002, pp. 3771-3780.

${ }^{19}$ Zhang, N., Chao, D. F., and Sankovic. J. M., "Two Basic Modes of Bubble Groeth and Determination of Departure Diameters in Pool Boiling," AIAA Meeting Papers on Disc [CD-ROM], Section 142-TP-10, 983 Reno, NV, 2006. 